\title{
OSCILACIONES NACIONALISTAS: DEL ETHOS GANDHIANO AL NACIONALISMO HINDÚ EN LA TRAYECTORIA POLÍTICA DE VALLABHBHAI PATEL
}

\author{
BEATRIZ MARTÍNEZ SAAVEDRA \\ E ISHITA BANERJEE \\ El Colegio de México
}

La filosofía y la política de Gandhi han sido objeto de análisis de numerosos trabajos importantes. Este ensayo se propone explorar las ideas de Gandhi desde una perspectiva distinta; es decir, a la luz de su percepción y aprehensión por Vallabhbhai Patel (también conocido con el título de sardar Patel), un líder importante del Partido Nacional del Congreso y uno de los seguidores clave de Gandhi. Ambos, Gandhi y Patel, provenían de Gujarat en India occidental, región donde Patel le dio liderazgo a los movimientos inspirados por Gandhi. El propósito es examinar cómo las ideas y las prácticas de Gandhi se prestaban a distintas y contradictorias aprehensiones y aplicaciones. Si los seguidores subalternos de Gandhi creían en su coraje así como en sus poderes mágicos y milagrosos y lo convirtieron en el mahatma, ${ }^{1}$ Vallabhbhai Patel aplicó la mezcla de lo religioso y lo político en el pensamiento de Gandhi a la retórica del nacionalismo (cultural) hindú; algo a lo que Gandhi siempre se opuso.

Este artículo procederá en dos pasos. Primero, destacará someramente los elementos importantes en las ideas y las prác-

Este artículo fue recibido por la dirección de la revista el 7 de enero de 2014 y aceptado para su publicación el 4 de febrero de 2014.

${ }^{1}$ Shahid Amin, "Gandhi como Mahatma: Distrito de Gorakhpur, UP Oriental, 1921-1922”, en Saurabh Dube (coord.), Pasados poscoloniales. Colección de ensayos sobre la nueva historia y etnografía de la India, México, El Colegio de México, 1999, pp. 345-401. 
ticas políticas y la filosofía de Gandhi, para luego desentrañar cómo se prestaban a distintas percepciones. Segundo, se concentrará en el nacionalismo hindú en general y en la figura de Vallabhbhai Patel en particular, en un intento por entender la interrelación de la lucha nacionalista india por la independencia del gobierno colonial y sus corrientes nacionalistas hindúes, representadas por Gandhi y Patel respectivamente. En lugar de ofrecer una discusión detallada de la lucha nacionalista, el artículo se referirá a ella como el contexto en el cual las ideas y las políticas de Gandhi y Patel fueron desarrolladas e implementadas.

Sin entrar al análisis de las concepciones de nación y nacionalismo, indicaremos solamente una característica del nacionalismo que tiene suma importancia en el tema de este artículo. El nacionalismo, se ha señalado, tiene vínculos con dos tendencias intelectuales distintas, incluso algunas veces opuestas: por un lado, abreva de la herencia del liberalismo y el secularismo e intenta establecer un sistema de gobierno basado en la autodeterminación sin distinción de clase, credo o color; por otro lado, está íntimamente ligado al "sistema de significados políticos" asociados con símbolos de la "comunidad”. ${ }^{2}$ Tales símbolos pueden ser de religión, casta, región o lenguaje. Pero la expresión habitual de comunidad implica una "comunalidad" percibida, una comunalidad con el potencial de transformar a una diversidad de personas en un cuerpo colectivo.

Además, como los símbolos no tienen significado intrínseco sino que funcionan como algo que ayuda a la gente a "crear sentido", 3 promueven esta percepción de comunalidad en formas discretas; por lo tanto, no es difícil entender que puede recurrirse fácilmente a la "religión" como un medio para crear la "comunalidad" que pueda forjar una comunidad. Este artículo tocará la percepción de Gandhi acerca de la sociedad india y sus prácticas para crear una comunalidad —el hecho de que aceptara la diferencia pero que esta comunalidad no se basara en la distinción entre religión o región-y rastreará las transformaciones que Patel hacía del colectivo "hindú".

\footnotetext{
${ }^{2}$ Burton Stein, A History of India, Sussex, Willey-Blackwell, 2010, p. 274.

${ }^{3}$ Anthony P. Cohen, The Symbolic Construction of Community, Londres, Routledge, 1989, p. 16.
} 


\section{La política de Gandhi}

Gandhi ofrece un ejemplo ilustrativo del pensamiento que considera la inseparabilidad de lo religioso y lo político. "Política sin moral”, declaró, tiene que ser evitada. "[N]o la religión de los supersticiosos y los ciegos”, aclaró, ni una religión que odia y riñe, sino una religión de tolerancia universal. ${ }^{4}$ Su idea de swa (propio) raj (autogobierno) y su herramienta para alcanzar este verdadero autogobierno, satyagraha (fuerza espiritual), estaban basados en la moral. Su muy discutido trabajo Hind Swaraj pone mucha atención al dharma, que para Gandhi consistía "no en la religión hindú, mahometana o zoroastriana”, sino en la religión que está dentro o sostiene a todas las religiones. ${ }^{5}$ Para él, la civilización moderna estaba haciendo a India "irreligiosa", tal como había hecho irreligiosa a Gran Bretaña.

Para Gandhi, los significados de swaraj de ninguna manera se referían a la mera emancipación del aparato colonial; evocaban una libertad que sólo podía alcanzarse "a través del gobierno ético del ser y la práctica rigurosa de la autodisciplina en la búsqueda por la verdad". ${ }^{6}$ Gandhi no equiparaba el autogobierno con la independencia o con el fin del imperio; esto le hacía "concordar extrañamente" con el imperio.? Para Gandhi, swaraj tenía estrechos vínculos con abimsa (no violencia) y satyagraba (fuerza espiritual). El anhelo y la insistencia (agraha) por satya (verdad), que es constitutiva del ser, "resalta la naturaleza activa de la no violencia". ${ }^{8}$ Mientras que Gandhi como líder del Congreso Nacional indio defendía el "swaraj político", también insistía en que el otro swaraj sólo podía ser alcanzado por medio

${ }^{4}$ Gandhi, "May God Help", Young India, 24 de noviembre de 1924, citado en Ajay Skaria, "La religión de Gandhi”, en Saurabh Dube e Ishita Banerjee (coords.), Otras modernidades: historias, culturas, identidades, México, El Colegio de México, 2011, p. 161.

${ }^{5}$ M. K. Gandhi, Hind Swaraj or Indian Home Rule, Ahmedabad, Navajivan Publishing House, 1938, capítulo 8.

${ }^{6}$ Tim Pratt y James Vernon, “'Appeal from This Fiery Bed...': The Colonial Politics of Gandhi's Fasts and their Metropolitan Reception”, Journal of British Studies, vol. 44, núm. 1, 2005, p. 95.

${ }^{7}$ Uday S. Mehta, "Patience, Inwardness, and Self-Knowledge in Gandhi's Hind Swaraj”, Public Culture, vol. 23, núm. 2, 2003, p. 425.

${ }^{8}$ Ajay Skaria, "Immeasurable Equality: Gandhi and the Gift of Religion", manuscrito inédito. 
de satyagraha. Este swaraj no era únicamente diferente, sino opuesto al "swaraj político". El swaraj político sólo proporcionaba el pretexto o punto de partida para el otro swaraj.

Satyagraba, por su parte, fue concebido como dharma, religión, fe, moral y ética. En los escritos de Gandhi, dharma propugna por la igualdad con el inferior, igualdad que no hace del inferior un superior; una igualdad alcanzada por medio de una genuina renuncia de sí mismo, una igualdad condicionada/precedida por el amor, la humildad y el respeto mutuo. Esta renuncia voluntaria posibilitaba una resistencia diferente a la dominación, en la que la renuncia misma se convertía en un modo de rechazar la subordinación, "de dar y recibir una libertad y una igualdad que están latentes en el acto mismo de renunciar".9 En este sentido, los satyagrabi (los que practican el satyagraba) no necesitan tomar las armas; al promulgar la "renuncia (o sumisión) sin subordinación” abandonan la libertad inherente al ejercicio cotidiano de soberanía, y ese abandono a su vez abre otra libertad. Cuando ellos se rinden, los satyagrabi entregan al otro sólo sus diferencias respecto del otro; de este modo, "se trastocan a sí mismos y al otro; este trastorno es swaraj”. ${ }^{10} \mathrm{Al}$ mismo tiempo, sentía que el imperio y la civilización enturbiaban la posibilidad de desarrollar el autoconocimiento que había de hacer posible el autogobierno.

Lo que subyace en el fondo de la noción moralizadora de nación de Gandhi es la creencia de que India podía ser libre sólo cuando sus individuos hubieran sucumbido a la búsqueda por la verdad y la no violencia (abimsa) y se reformaran y aceptaran el sarvodaya (servicio incondicional-desinteresado). Sólo entonces la satyagraha (fuerza espiritual), "el arma fuerte" de Gandhi, sería efectiva como forma de lucha. Un auténtico satyagrabi tenía que ser puro y saludable de cuerpo y mente; tendría control total sobre sus sentidos, su pasión y su "lujuria”, y se comprometería en la búsqueda de la verdad, lo cual marcaría el camino hacia swaraj.

Por lo tanto, queda claro que el vocabulario político de Gandhi estaba imbuido de símbolos religiosos: votos, promesas 
y juramentos tenían un lugar importante en sus escritos y en sus prácticas de la política. Al mismo tiempo, tal afirmación de política religiosa iba de la mano con una afirmación del secularismo. La idea de nación de Gandhi era inclusiva, y lo que él llamaba religión en realidad puede ser leído como "ética en el más amplio e inclusivo sentido de la palabra". ${ }^{11}$ La insistencia de Gandhi en que no podía haber "ninguna política sin religión” era una "poderosa forma de apropiación de valores éticos para fundamentar la política”. ${ }^{12}$

Un análisis temprano del encanto de Gandhi había indicado que su "perceptiva visión de la estructura de la sociedad en India", junto con "una profunda percepción de las lealtades sociales del individuo y cómo éstas pueden ser invocadas a la acción política”, estaban en el centro del éxito de Gandhi. ${ }^{13}$ Mientras que líderes nacionalistas que precedieron a Gandhi asumieron la existencia de una sociedad política homogénea, que constreñía sus esfuerzos para organizar un movimiento político de masas, Gandhi, de una manera que llama la atención, compartía la visión de los administradores británicos de que India era una vaga constelación de clases, comunidades y grupos religiosos. Sin embargo, en lugar de tomar esto como una desventaja de la sociedad política, él, de manera efectiva, apeló a las lealtades de distintos grupos y trajo consigo una movilización de masas sin paralelo en la historia de India. ${ }^{14}$

Gandhi, como un líder político, argumenta Partha Chatterjee, provenía no "de lo razonable de su programa", sino de "reivindicaciones morales de valor personal y sacrificio y una patente fidelidad a la verdad". Tales reivindicaciones le conferían la total lealtad de su gente, ${ }^{15}$ y sobre la base de estas reivindicaciones morales Gandhi llamó a las personas repetidamente a hacer votos, promesas y juramentos (vrats y yama-

${ }^{11}$ Skaria, "La religión de Gandhi", op. cit., p. 166.

${ }^{12}$ Idem.

${ }^{13}$ Ravinder Kumar, “Class, Community or Nation? Gandhi's Quest for a Popular Consensus in India”, Modern Asian Studies, vol. 3, núm. 4, 1969, p. 361.

${ }^{14}$ Idem.

${ }^{15}$ Partha Chatterjee, "Gandhi and the Critique of Civil Society", en Ranajit Guha (ed.), Subaltern Studies III: Writings on South Asian History and Society, Nueva Delhi, Oxford University Press, 1984, p. 188. 
niyamas) durante cada una de sus campañas. Dharma, o la religión, supone el cumplimiento del voto o las promesas o "la conversión de ser humano en un animal que hace promesas; en una figura que se distingue por la repetición y el retorno". ${ }^{16}$ Con esta breve explicación de la filosofía y la política de Gandhi, pasemos a Gujarat, tierra natal de Gandhi, y a su más importante discípulo ahí, Vallabhbhai Patel.

\section{Vallabhbhai y la lucha nacionalista en Gujarat}

Los estudios sobre Vallabhbhai Patel han oscilado entre algunas tendencias bien definidas; por un lado, están las exploraciones de su lucha anticolonial y la organización de las satyagrabas o campañas nacionalistas en el marco del liderazgo de Gandhi; igualmente, están los estudios que poseen un tono laudatorio, al evaluar a Patel como un héroe nacional. ${ }^{17}$ En esta perspectiva, el desempeño de Vallabhbhai es observado a la luz de su llamado a la unidad de hindúes y musulmanes hacia finales de la década de 1930 y, también, de su exitosa gestión en el acceso a India de los estados principados luego de la partición.

En contraste, al otro lado del espectro están los análisis que lo etiquetan como un nacionalista hindú equiparable a los ideólogos más recalcitrantes de esta corriente; en particular, por su actitud antiislámica en la década de $1940 .{ }^{18}$ Si bien tales aspectos son facetas en la postura ideológica de Vallabhbhai, es necesario dilucidar las discontinuidades siguiendo el periplo de su actuación política, porque la identidad de Patel se vio transfigurada por el devenir histórico, que moldeó las transformaciones que se registraron en el influyente político. ${ }^{19}$

${ }^{16}$ Skaria, "La religión de Gandhi”, op. cit., p. 160.

${ }^{17}$ Balraj Krishna, India's Bismarck, Sardar Vallabhbhai Patel, Mumbai, Indus Source Book, 2007.

${ }^{18}$ Parita Mukta, "On The Political Culture of Authoritarianism”, en Ghanshyam Shah, Mario Rutten y Hein Streefkerk (eds.), Development and Deprivation in Gujarat, Nueva Delhi-Thousand Oaks, Sage Publications, 2002, pp. 59-73.

${ }^{19}$ Estudios recientes han prestado mayor atención a la postura política cambiante de Vallabhbhai Patel en virtud de los acontecimientos, que le hacían reformular sus estrategias políticas. Véase Arafaat Valiani, Militant Publics in India. Physical Culture and Violence in the Making of a Modern Polity, Nueva York, Palgrave-Macmillan, 2011. 
Para ubicarlo en su justa dimensión, contamos con volúmenes que recopilan diversos documentos del personaje; por ejemplo, sus cartas y algunos de sus discursos. Asimismo, las notas periodísticas que describen sus actividades también son relevantes en la reconstrucción histórica de Patel. Por otro lado, los documentos de archivo utilizados, como los reportes confidenciales del gobierno sobre organizaciones e individuos que podían representar algún inconveniente para la paz social, arrojan mucha luz sobre la evolución ideológica del político. En conjunto, los textos recopilados y los explorados en el archivo integran un nutrido corpus que nos aproxima de manera directa a su pensamiento y transformación.

Vallabhbhai Patel nació en el pequeño poblado de Nadiad, en 1875, y fue educado en el seno de una familia patidar de Charotar. Los patidar o patel eran un grupo dominante que disfrutaban de importante influencia política; por ejemplo, a veces fungían como matadars, es decir, los que recolectaban la renta en su linaje, lo cual les permitía tener dominio sobre los campesinos más pobres..$^{20}$ De los patidar se dice que eran reconocidos por su independencia y liderazgo pero también que a veces podían ser arrogantes y tener cierto sentido de superioridad frente a otras comunidades. ${ }^{21}$

Vallabhbhai se formó en leyes y litigó en Godhra y Borsad junto con su hermano mayor, Vithalbhai Patel, y aunque había logrado reunir dinero para ir a Londres a estudiar, cedió a su hermano el privilegio de ir primero. Posteriormente, completó su formación en Londres, de 1910 a 1913, y a su regreso ejerció en Ahmedabad en vez de Bombay, que era una ciudad más lucrativa. Como consejero municipal de Ahmedabad de 1917 a 1922, Vallabhbhai ya desafiaba a la autoridad británica encarnada en John Shillidy, el comisionado municipal británico, de quien se quejaba por su comportamiento incivilizado y sus actos de corrupción.

El nacionalismo que Vallabhbhai Patel desarrolló por esos años era de tipo anticolonial, y se sintetizaba en su idea de que

${ }^{20}$ David Hardiman, Peasant Nationalists of Gujarat. Kheda District 1917-1934, Delhi, Oxford University Press, 1981, p. 37.

${ }^{21}$ Rajmohan Gandhi, Patel. A Life, Ahmedabad, Navajivan Publishing House, 1990, p. 4. 
el régimen colonial en "India [era] un jardín de esclavos: algunos eran grandes, otros pequeños, algunos valientes pero todos son esclavos". ${ }^{22}$ De este modo, Patel admiraba la actitud desafiante hacia los oficiales británicos y fue esta cualidad que apreció en Gandhi lo que le hizo allegarse a él.

A partir del regreso de Gandhi de Sudáfrica y su llegada a Ahmedabad en 1915, Gujarat adquirió una posición preponderante en la lucha nacional. Vallabhbhai mostró su cercanía a Gandhi al organizar campañas que siguieron el método desarrollado por su mentor - es decir, la satyagraba- en Kheda en 1918, Borsad en 1923, y Bardoli en 1928, antes y después del movimiento de No Cooperación Califato de 1921-1922, liderado por Gandhi y el Congreso. Estas campañas identificaban al régimen británico como el objetivo que debía vencerse en la lucha nacional, aunque, para Gandhi, como se ha referido, swaraj no era un simple autogobierno basado en la liberación del régimen colonial sino una liberación sustentada en una base ética.

Vallabhbhai Patel fungió como el brazo derecho de Gandhi en las campañas que realizó en su natal Gujarat y adquirió un gran estatus como luchador social..$^{23}$ Merece la pena detenerse en los términos en que Patel emprendió estas campañas, pues en las tres satyagrahas se perfiló como un gran organizador de masas con la habilidad para motivar o presionar a los campesinos y afianzar la solidaridad con su causa. ${ }^{24}$

En la articulación de las satyagrahas, Gandhi representaba la autoridad moral y sardar Patel, el brazo fuerte. ${ }^{25}$ En otras palabras, en el binomio Gandhi-Vallabhbhai, el primero proporcionaba una dimensión ética a las campañas, y el segundo aplicaba métodos severos para lograr involucrar a los diversos

${ }^{22}$ Archivo Estatal de Maharashtra (AEM), Home Department Special Branch (HDSB), exp. 800(98), 1935, p. 77: "Copy of the Confidential Report dated 5/1/35 del S.H. S.I.S.S. Mankad, Ahmedabad. Activities of Vallabhbhai Patel in Gujarat after his release from jail in July 1934".

${ }^{23} \mathrm{Idem}$.

${ }^{24}$ En este sentido, Gandhi consideraba a Vallabhbhai un buen "agitador”. Carta de Gandhi a Mahadev Desai, 22 de septiembre de 1929. M. K. Gandhi, The Collected Works of Mahatma Gandhi, Nueva Delhi, Ministry of Information \& Broadcasting, Government of India, 1970, p. 453.

${ }^{25}$ Howard Spodek, "Sardar Vallabhbhai Patel at 100”, Economic Political Weekly, vol. 10, núm. 50, 1975, p. 1928. 
sectores aunque mostraran cierta renuencia; métodos que por coercitivos no se ceñían ciertamente al código gandhiano. En esta tesitura tuvo una gran autonomía para actuar como mejor consideraba que podía consolidarse el apoyo de los grupos sociales. Proveniente de un contexto patidar en que los miembros estaban habituados a ejercer cierta dominación sobre grupos más vulnerables, Vallabhbhai utilizó técnicas de presión como el boicot y el ostracismo a los miembros de la casta que no cooperaban. Gandhi discrepaba con ejercer algún tipo de presión social en las satyagrahas pues esto no coincidía con su noción de abimsa o no violencia.

En claro contraste, la visión de abimsa del sardar era un tanto ambigua porque "él mismo no estaba seguro de ser un partidario de abimsa". ${ }^{26}$ Para Vallabhbhai, "la no violencia no significa debilidad o timidez. Debía haber determinación y valentía en la no violencia y a nosotros nos falta mucho en este sentido". ${ }^{27}$ Sus métodos más militantes de movilización política privilegiaban el compromiso de tomar acciones en la práctica. ${ }^{28}$ Congruente con su percepción, la fuerte presión psicológica y social ejercida sobre los campesinos para forzarlos a unirse a las campañas era necesaria en la lucha nacional por un ideal más elevado; asimismo, consideraba que la presente condición de los campesinos era en mucho su responsabilidad al ser omisos en luchar por lo que les correspondía. Así, Patel expresaba: "La razón de [la] pobreza [de los campesinos] es que no conocen su patria. Han perdido el verdadero heró́smo [...] El verdadero espíritu Rajput está extinto y se ha ido". ${ }^{29}$ Esto implicaba que la no violencia no debía usarse como pretexto para someterse; una idea casi completamente opuesta a la de Gandhi, de "sumisión sin subordinación". En efecto, Vallabhbhai parecía estar de acuerdo con la idea de estar físicamente preparados para oponerse a los enemigos. La proliferación de akhadas o gimnasios durante este periodo evidenciaba la convicción entre

${ }^{26}$ Gandhi, Patel. A Life, op. cit., p. 70.

${ }^{27}$ AEM, HDSB, exp. $800(98)$, 1935, op. cit.

${ }^{28}$ Valiani, Militant Publics in India, op. cit., p. 146.

${ }^{29}$ Oficina de Registros de Baroda (ORB), Confidential Documents (CD), Huzur Political Office (HPO), fólder 5, exp. 130: "English abstract of the speech delivered by Mr. Vallabhbhai J. Patel at Kurai in the Karjan taluka on 30-11-1937. Speeches delivered by political agitators". 
diversos sectores de desarrollar la capacidad física que rindiera sus frutos en la acción.

En este sentido, uno de los akhadas más célebres fue el del profesor Manekrao, en Baroda, ${ }^{30}$ en el que no sólo se impartía educación física y “defensa” personal sino que también se prestaba para que políticos extremistas, que visitaban Gujarat, dieran discursos sobre el nacionalismo. ${ }^{31}$ Vallabhbhai Patel también estuvo entre los conferencistas del akhada de Manekrao, pero fueron miembros de la Hindu Mahasabha _organización que promovía los valores culturales hindúes- quienes persistieron en la tarea de establecer akbadas en diversas partes de Gujarat con el fin de terminar con la supuesta emasculación hindú, que los tenía subyugados a británicos y musulmanes. La proliferación de los akhadas fue un hecho reprobado por el gandhismo, que lo veía como un franco alejamiento de la filosofía de la no violencia. Vallabhbhai, en contraste, no refirió estar en desacuerdo con las actividades de tales centros y, más aún, asistió a ellos durante sus campañas políticas.

\section{Comunidad, "comunalidad" y distintas connotaciones de swaraj}

La idea de Vallabhbhai de unidad comunitaria ha sido un aspecto polémico en su trayectoria política. Si bien Vallabhbhai hacía en sus campañas un llamado general a unirse a la lucha nacional, los musulmanes no se sentían muy identificados con una retórica fundamentalmente hindú. En este sentido, aunque varios autores han destacado el esfuerzo del sardar por unificar a las comunidades, ${ }^{32}$ lo cierto es que para él no era un asunto imperativo; de hecho, para Patel, la unidad comunitaria era un aspecto más bien marginal en su discurso político en el periodo

${ }^{30}$ ORB, CD, HPO, Catálogo de la Oficina de Registros de Baroda 21, exp. 36, p. 3: "Jummadada Gymnastic Institute of Mankekrao".

${ }^{31}$ Por ejemplo, Savarkar, el fundador de la ideología excluyente del Hindutva, estuvo ahí. HPO, CD, ORB, fólder 6, exp. 140, p. 294: "Confidential fortnightly report for the period ending $26^{\text {th }}$ April 1946. Fortnightly confidential reports from $12^{\text {th }}$ August 1945-48".

${ }^{32}$ Rafiq Zakaria, Sardar Patel and Indian Muslims. An Analysis of his Relations with Muslims before and after India's Partition, Bombay, Bharatiya Vidya Bhavan, 1996. 
de las satyagrabas, pues "Gujarat no [tenía] mucho que hacer en relación con la unidad hindú-musulmana en comparación con otras provincias; así que Gujarat tiene mucho margen para gastar sus energías en otro programa”. ${ }^{33}$ Esta aseveración no era necesariamente infundada, así lo confirman los reportes sobre disturbios que marcan un incremento de la violencia comunal hacia 1924, es decir, después de la satyagraba de 1923.

Durante el movimiento por el Califato, Vallabhbhai $\mathrm{Pa}-$ tel se había mostrado poco entusiasmado al igual que otros miembros del Congreso, pues las demandas del movimiento les parecían un tanto alienantes siendo ellos hindúes. ${ }^{34}$ Por el contrario, Gandhi con todo entusiasmo pregonaba que gustosamente pospondría el swaraj si hubiera progresos respecto al Califato. ${ }^{35}$ La noción polisémica de swaraj era en sí problemática; con tantos sectores incluidos en el movimiento de Gandhi, cada quien entendía swaraj en una forma diferente. ${ }^{36}$ Gandhi se refería a swaraj, según se esbozó en líneas anteriores, como un gobierno con una dimensión ética y no necesariamente como la emancipación del régimen colonial.

Pero en el entendimiento de swaraj como autogobierno, $\mathrm{Pa}$ tel no comprendía cómo podían luchar por los musulmanes de Arabia, Palestina, Siria e Iraq; a él le hacía más sentido lograr primero la emancipación de los británicos y luego pelear por otros. ${ }^{37}$ A pesar de sus reservas, el sardar apoyó el movimiento y emitió una convocatoria para "ayudar a proteger el islam brindando todo tipo de ayuda posible a los musulmanes y mostrar plena fe en la bondad de la comunidad". ${ }^{38}$

${ }^{33}$ Vallabhbhai Patel, The Collected Works of Sardar Vallabhbhai Patel, ed. P. N. Chopra, vol. I: 1918-1925, Delhi, Konark Publishers, 1991, p. 235.

${ }^{34}$ Hamza Alavi ha hecho notar que el movimiento por el Califato apelaba a las premisas más anquilosadas del islam, el cual consideraba al califa como el monarca y el líder religioso, a diferencia del islam "original", que distinguía entre los dos dominios como esferas separadas. Véase Hamza Alavi, "Ironies of History: Contradictions of the Khilafat Movement", en Mushirul Hasan (ed.), Islam, Communities and the Nation: Muslim Identities in South Asia and Beyond, Nueva Delhi, Manohar, 1998, p. 35.

${ }^{35}$ Krishna, India's Bismarck, Sardar Vallabhbhai Patel, op. cit., p. 81.

${ }^{36}$ V'éase Amin, "Gandhi como Mahatma", op. cit.

${ }^{37}$ Indulal Yagnik, Atmakatha, citado en Achyut Yagnik y Suchitra Sheth, The Shaping of Modern Gujarat: Plurality, Hindutva and Beyond, Nueva Delhi, Penguin, 2005, pp. 207-208.

${ }^{38}$ Zakaria, Sardar Patel and Indian Muslims, op. cit., p. 8. 
Asimismo, el lenguaje religioso que Vallabhbhai conocía era el de su propio contexto cultural; por lo tanto, utilizaba vocabulario del hinduismo para describir el movimiento, lo cual no atraía a los musulmanes. A diferencia de Gandhi, Vallabhbhai no concebía el dharma con una dimensión universalista; la "comunalidad" que Patel articulaba entre los hindúes se hacía a través de apelar al hinduismo, en su religiosidad particular. La lucha nacional era comparada con la pelea entre Ram y Ravana de la épica del Ramayana; también se refería al dharma como el deber moral de unirse al movimiento nacional o hablaba de shakti o energía para ilustrar la fuerza que debía tenerse para combatir a los ingleses. ${ }^{39}$ En tanto que Gandhi concebía la lucha nacional como un deber sagrado y no visualizaba la política separada de la religión; su visión era el establecimiento del Ramraj o el reino de Ram, no en una dimensión sectaria sino entendido como el "gobierno de Dios", por medio del cual se establecerían valores humanitarios. ${ }^{40}$ Vallabhbhai hacía notar que el "Mahatma había empezado este movimiento no sólo contra el gobierno sino contra nosotros mismos. Tenemos que purificar el gobierno y también hacer autopurificación". ${ }^{41}$ De esta manera, el movimiento nacional brindaba la posibilidad de expiar los "pecados" además de ejercer oposición al gobierno británico. Era una campaña para resolver social y personalmente asuntos éticos.

Por otro lado, la estrategia discursiva de Vallabhbhai fue fundamental en la intimidación e inclusión de los potenciales activistas al aludir a imaginarios religiosos que daban una responsabilidad ética a su participación. ${ }^{42}$ Existen por lo menos tres aspectos que dan forma a la retórica de Vallabhbhai: fervor nacional, ética y religiosidad. Al mezclar estos elementos, Patel

${ }^{39}$ AEM, HDSB, exp. 750(86)I, 1930-1931, pp. 47-49: "Independence and civil defense movement. Extracts of speeches of Vallabhbhai Patel and other Congress discouraging the payment of land revenue in Gujarat district".

${ }^{40}$ Irfan Habib, "Gandhi and the National Movement", Social Scientist, vol. 23, núm. 4, 1995, p. 8.

${ }^{41}$ AEM, HDSB, exp. 800(98), 1935, op. cit.

${ }^{42} \mathrm{El}$ discurso de Patel y otros líderes en Bardoli es explorado con detalle por Ghanshyam Shah en "Traditional Society and Political Mobilization: The Experience of Bardoli satyagraha (1920-1928)", Contribution to Indian Sociology, vol. 8, núm. 89, 1974, pp. 89-107. 
esperaba apuntalar la identificación y el apoyo subsecuente para la causa nacionalista; hacía el llamado a "desobedecer las órdenes inmorales del gobierno" 43 y resistir a las autoridades sin importar la adversidad que franquearan, porque "mientras mayor la tiranía que Gujarat sufriera, mayor su santificación". ${ }^{44}$

La idea de moral o ética es relevante en la lucha nacional que Vallabhbhai vislumbra. La salvación nacional e individual se lograría a través de un comportamiento ético intachable como individuos. Esta creencia hace que el discurso de Vallabhbhai esté permeado por un ingrediente normativo; entre otras cosas, la limpieza, el vestir khadi, el no al matrimonio infantil, no al ocio, no a las discusiones, la no discriminación de los intocables, eran algunos asuntos que Vallabhbhai promovía, no sólo en paralelo con la lucha nacional sino también como aspecto fundamental para lograrla; es decir, una regeneración moral de los individuos. Otras actividades de las que había que cuidarse eran el consumo de opio y la ingesta de alcohol ya que, en su opinión, eran "vicios que debían ser condenados por todos". ${ }^{45}$ En consonancia con esta idea se fundaron sabhas antialcohol en 1929, cuyo propósito era la abolición del consumo de droga y alcohol. ${ }^{46}$ De este modo, el activismo de Vallabhbhai en la lucha nacional tiene muchos elementos de coerción. Pero el mensaje era claro, la lucha nacionalista demandaba sacrificios de toda índole.

Después de la época de las satyagrahas, los años de 1927 a 1929 se tornaron bastante inciertos en términos de hostilidad comunal en varios lugares de Gujarat, como Godhra y Surat. Líderes de organizaciones hindúes, como la Hindu Mahasabha, trabajaban con una agenda común para consolidar los valores hindúes entre las minorías de distinta filiación religiosa y también impulsaban las hostilidades entre sectores de hindúes y musulmanes. En 1927, por ejemplo, un representante local de la Hindu Mahasabha, llamado Vamanrao Mukadam, promovía

${ }^{43}$ Patel, The Collected Works, op. cit., vol. I, p. 14.

${ }^{44}$ Memorial, Museo y Biblioteca Nehru, Private Papers of K. M. Munshi, rollo 19, exp. 15: "K. M. Munshi and Vallabhbhai Patel's Correspondence”.

${ }^{45}$ AEM, HDSB, exp. 800(98), 1935, op. cit.

${ }^{46}$ Young India (1919-1931), “M. K. Gandhi, 'Prohibition Campaign'”, vol. XI, 23 de mayo de 1929, p. 171. 
la discordia entre las comunidades en Godhra. Al participar en una procesión blandía amenazadoramente un revólver para intimidar a los musulmanes que se quejaban de la música frente a la mezquita, al tiempo que azuzaba a los participantes de la procesión a continuar con la música. ${ }^{47}$ Mukadam también era un relevante miembro del Congreso y, en 1917, había organizado la Conferencia Política de Gujarat, a la que habían asistido Gandhi, Jinnah y Tilak. ${ }^{48}$ Según información de un trabajador social, seguidor de Gandhi, Vallabhbhai Patel estaba en buenos términos con Mukadam y ambos se encontraban a menudo para hablar sobre los asuntos entre hindúes y musulmanes. Por su "claridad al hablar, [Mukadam] permaneció como amigo cercano de sardar Patel de por vida". ${ }^{4}$

Acaso por esta cercanía, sardar nunca hizo alguna condena contundente hacia la Mahasabha y su activismo, ya que miembros del Congreso y la propia organización hindú tenían relaciones cordiales en diferentes momentos. En este sentido, es interesante resaltar que en los disturbios de 1927-1928, en Godhra, aunque ocurrieron en un periodo muy cercano a una de las satyagrahas más emblemáticas de Vallabhbhai, la de Bardoli, él nunca reprobó la incitación a la violencia encabezada por Mukadam, y es difícil pensar que no se hubiera enterado de los acontecimientos.

Esto nos lleva a cuestionar hasta qué punto los miembros del Congreso congeniaban plenamente con la noción gandhiana de unidad comunitaria y la no violencia y, asimismo, cuál era el entendimiento o las interpretaciones que otros personajes políticos hacían de las concepciones de Gandhi. Tanto Vallabhbhai como Mukadam eran miembros del Congreso. Visiblemente, Mukadam omite de una manera abierta la abimsa de Gandhi y se va al otro lado del espectro al conminar a la violencia, lo cual nos reafirma que el afán del Congreso por incorporar a diversos sectores abrió la puerta a tendencias extremistas.

${ }^{47}$ AEM, HDSB, exp. 355(59)A vIII, 1927, p. 1: "Extract from the confidential weekly letter of the District Magistrate, Panch Mahals, for the week ending 24 $4^{\text {th }}$ August 1927, Home Department Special Note, W. S. Mukadam”.

${ }^{48}$ Yagnik y Sheth, The Shaping of Modern Gujarat, op. cit., p. 214.

${ }^{49}$ Arun Vaghela, "Vamanrao Mukadam: the Lion of PanchMahal", en Arun Vaghela, Anvesna Itihasna Lekhono Sangrah, Gandhinagar, Gujarat Sahitya Akademi, 2001, pp. 93-100. 
Asimismo, los políticos realmente cercanos a Gandhi, como Vallabhbhai, tampoco parecían ceñirse demasiado a los valores gandhianos; esto se ve en su indiferencia hacia la violencia de Mukadam, pues sus llamados a la no violencia no trascendían la retórica cuando se trataba de oponerse a los métodos de movilización de extremistas.

\section{La partición y el sentimiento antiislámico}

La década de 1940 marca un cambio en su "apego" a los principios de Gandhi y, asimismo, en las consideraciones de unidad de Vallabhbhai Patel. Si antes creía innecesario hacer una convocatoria intensa por esta causa, para este periodo lo cree ineludible ante la idea de la creación de Pakistán posicionándose cada vez más fuerte en la escena política. En sus inicios como luchador social, Vallabhbhai identificaba a la administración británica como el enemigo a vencer; en este periodo también ve como un lastre a la Liga Musulmana, que además iba ganando terreno entre los adeptos al islam. En este contexto le parecía desesperante que alguien como Jinnah, líder de la Liga y musulmán no practicante, tuviera tanta influencia en la comunidad islámica, a diferencia del líder musulmán del Congreso Abul Kalam Azad, y del propio Gandhi, que había perdido el apoyo de la gran masa de musulmanes desde el llamado a terminar el movimiento por el Califato. Sus discursos tratarían de dar un sentido de unidad a las comunidades en India; así expresaba: "India es una e indivisible [...] Los musulmanes tienen sus raíces en India. Sus lugares sagrados y sus centros culturales están ubicados en India. No sé qué es lo que harían en Pakistán y no pasaría mucho tiempo antes de que quisieran volver"..$^{50}$

En su intento por mantener la unidad de India, Vallabhbhai trataba de fortalecer la imagen del régimen británico como el enemigo común, y hacía referencia a India como un "jardín de esclavos" donde todos debían unirse para terminar con esa esclavitud. Refería que la historia les había enseñado que la condición fragmentada entre los grupos sociales había he-

${ }^{50}$ Patel, The Collected Works, op. cit., vol. XII, p. xvi. 
cho que sucumbieran ante los invasores extranjeros. "Nuestros conflictos mutuos, peleas y desconfianzas -mencionaba - han sido en el pasado la causa de nuestro desplome y el haber caído presos de la dominación extranjera un sinnúmero de veces". ${ }^{51}$

La actitud de unidad que Vallabhbhai refleja en público contrasta con su suspicacia personal por los musulmanes, lo cual hace que le exprese a Gandhi que "las maneras y costumbres de los musulmanes son diferentes. Ellos comen carne mientras nosotros somos vegetarianos. ¿Cómo vamos a vivir con ellos en el mismo lugar?”. A lo que Gandhi replicaba: "No señor. Los hindúes en conjunto no son vegetarianos, excepto en Gujarat. Casi todo hindú come carne en el Punjab, U. P. y Sind" ${ }^{52}$ De manera que aunque Vallabhbhai tiene un discurso público de existencia comunitaria conjunta, en realidad no se ve compartiendo los mismos espacios físicos con gente que le resulta problemática por sus hábitos alimentarios. Aunque al mismo tiempo y tratando de superar sus propios prejuicios advertía públicamente contra el hábito de "sospechar unos de otros", porque esto llevaba a la segregación. ${ }^{53}$ Esta postura conciliatoria fue redefinida con la partición de India. Las hostilidades que tal acontecimiento conllevó hicieron finalmente que Vallabhbhai tomara partido a favor de los hindúes; su discurso, entonces, subrayaba la "mucha enemistad entre las dos comunidades: en Calcuta, Lahore y Bombay, a donde quiera puede verse que se han formado mini-Pakistanes. Ningún hindú puede entrar a alguna localidad musulmana". ${ }^{54} \mathrm{El}$ alejamiento de Vallabhbhai de los principios gandhianos puede explorarse mejor a la luz de los hechos de la partición y su actuación en la integración de los estados principados.

Con la partición se les dieron tres opciones a los estados principados: unirse a India, acceder a Pakistán o permanecer independientes. Como ministro de Asuntos Internos, a Vallabh-

${ }^{51}$ Ibid., p. xiii.

${ }^{52}$ Zakaria, Sardar Patel and Indian Muslims, op. cit., p. 18.

${ }^{53}$ Ibid., p. 8.

${ }^{54}$ Vallabhbhai Patel, For a United India: Speeches of Sardar Patel, 1947-1950, Delhi, Publications Division, Ministry of Information and Broadcasting, Government of India, 1967, p. 158. 
bhai le correspondió hacerse cargo de la integración. Los casos más conflictivos fueron el de Junagadh, el cual, en una decisión de último minuto, decidió unirse a Pakistán; asimismo, el caso de Hyderabad, que decidió quedarse independiente con la posibilidad de unirse posteriormente a Pakistán.

Junagadh era un estado indio cuyo gobernante musulmán, nabab Mahabat Khan, había decidido acceder a Pakistán sin considerar que $80 \%$ de la población de Junagadh era hindú y sólo $20 \%$ era musulmán. El anuncio de Junagadh adhiriéndose a Pakistán tomó por sorpresa a Vallabhbhai en las noticias de periódico del 17 de agosto; Patel averiguó, en primer lugar, si Pakistán había aceptado la anexión..$^{55}$ Para el 24 de septiembre de 1947, Vallabhbhai ya había enviado tropas indias a las fronteras de Junagadh en espera de que Pakistán cancelara el acceso de Junagadh y de que se organizara un plebiscito. ${ }^{56}$ Más allá de la cuestión operativa es interesante reparar detenidamente en la retórica de Vallabhbhai a la luz de estos acontecimientos, porque ya ha dado un giro radical en su pasada tolerancia e inclusión de los musulmanes a la nación india. Su transformada actuación política le valió pasar a la posteridad como uno de los iconos del imaginario del Hindutva del periodo contemporáneo. ${ }^{57}$

Con la derrota del gobernante de Junagadh y su huida a Pakistán, Vallabhbhai visitó el lugar y dio un discurso en el Bahauddin College ${ }^{58}$ institución que estaría afiliada a la universidad de Sind, en Karachi, cuando Junagadh accediera a Pakistán..$^{59}$ En este discurso, Vallabhbhai informaba a la concurrencia del referéndum sobre el acceso de Junagadh y advertía a los musulmanes que fueran fieles a la unión india; asimismo, les dijo: "aquellos que quieran irse a Pakistán pueden hacerlo con sus propiedades". En dicha asamblea, Patel organizó un referéndum

${ }^{55}$ Para tener un panorama más amplio del episodio en su conjunto, véase idem.; Gandhi, Patel. A Life, op. cit.; y V. P. Menon, The Story of the Integration of the Indian States, Bombay, Orient Longman, 1969.

${ }^{56}$ Gandhi, Patel. A Life, op. cit., p. 436.

${ }^{57}$ Parita Mukta, "On The Political Culture of Authoritarianism", op. cit.

${ }^{58}$ The Bombay Chronicle, "We'll hand over Junagadh to provisional government alone' Sardar's reply to Pakistan: Token referendum favours Indian Union”, vol. Xxxv, núm. 268, 15 de noviembre de 1947, p. 9.

${ }^{59}$ Krishna, India's Bismarck, Sardar Vallabhbhai Patel, op. cit., p. 122. 
extraoficial donde la gente votó unánimemente por unirse a India. ${ }^{60}$

Ahora, en vez de hacer alusión a la unidad, Vallabhbhai advertía a los musulmanes que "si insistían en hablar de Pakistán, tendrían que seguir el ejemplo del nabab de Junagadh, quien había huido a Karachi. Los musulmanes habían pensado [...] que Pakistán sería el cielo en la Tierra, pero era el infierno". ${ }^{61}$ De este modo, cuando Vallabhbhai llegó a Junagadh, el 13 de noviembre, con la investidura de primer ministro de India, junto con N. V. Gadgil, ministro de Obras, Energía y Minas, una prioridad para él fue visitar el templo de Somnath, en Prabhas Patan, y anunciar su reconstrucción..$^{62}$ Así, se refiere que estaba visiblemente conmovido de encontrar el templo — que había sido alguna vez la gloria de India- ${ }^{63}$ tan destruido y dilapidado. De aquí que propusiera su reconstrucción para devolverle su antiguo esplendor. ${ }^{64}$

Evidentemente, esto era un asunto delicado en un momento donde las pasiones comunales estaban exacerbadas. Somnath era un templo de sensibilidad hindú específicamente, se suponía que era el lugar más sagrado después de Benarés (Varanasi), así que la decisión de su reconstrucción por altos mandos del gobierno transmitía el mensaje de supremacía del hinduismo sobre otros credos religiosos. Gandhi había percibido este mensaje y recomendaba que la reconstrucción del templo de ninguna manera se llevara a cabo con presupuesto del gobierno sino con contribuciones de la gente. Así, se concretó la reconstrucción y esta situación fue subrayada durante la ceremonia de instalación del ídolo de Somnath en $1951 .^{65}$ Sin embargo, que las autori-

${ }^{60}$ The Bombay Chronicle, “'We'll hand over Junagadh...”, op. cit.

${ }^{61}$ The Times of India, "Pakistan's interference in Junagadh; Sardar Patel's criticism”, vol. cIX, núm. 273, 15 de noviembre de 1947, p. 9.

${ }^{62} \mathrm{Idem}$.

${ }^{63}$ El templo de Somnath, se suponía, había sido destruido por un invasor musulmán (Mahmud de Ghazni) en el siglo XI, y para la primera mitad del siglo XIX ya iba cobrando forma en la mentalidad hindú como el recordatorio de la persecución del hinduismo a manos del islam. Romila Thapar hace una exploración histórica de cómo se convirtió este episodio en el símbolo de la hostilidad islámica, a pesar de que las diversas fuentes contemporáneas registran el hecho de maneras diversas o en ocasiones incluso ni siquiera se menciona. Consultar Romila Thapar, Somanatha, The Many Voices of a History, Nueva Delhi, Penguin, 2004.

${ }^{64}$ Menon, The Story of the Integration of the Indian States, op. cit., p. 141.

${ }^{65}$ The Bombay Chronicle, "Somnath", vol. Xxxix, núm. 13, 12 de mayo de 1951, p. 4. 
dades anunciaran la reconstrucción del templo y la posterior ceremonia inaugural a la que asistió el presidente, Rajendra Prasad, difundió el mensaje de un gobierno que patrocinaba la religión de la mayoría y marginaba la noción de Gandhi de una religiosidad universalista e incluyente. Esta actitud de Vallabhbhai Patel de formar una India fuerte y territorialmente cohesionada se ve reflejada en la coerción sobre principados "musulmanes" importantes, como Hyderabad, a acceder a la unión india. De la misma manera, Patel se enfocó en los testimonios de los refugiados hindúes llegados de Pakistán, quienes le habían relatado los horrores vividos, para privilegiar el restablecimiento de estos sectores por sobre los musulmanes que pasaban por situaciones adversas paralelas.

Todo lo anterior muestra que la transformación de Vallabhbhai se había completado, y de ser un "devoto" promotor de la unidad hindú-musulmana - como muchos de sus simpatizantes lo han visto-, en consonancia con la filosofía de Gandhi, se convirtió en un político que demandaba a los musulmanes proporcionar las evidencias de su lealtad hacia India, y si no podían hacerlo entonces les pedía mejor marcharse. Su exigencia de mostrar lealtad a India era efectiva, tanto que hubo organizaciones islámicas como la Jamiyat Muslims que declararon su decisión de disolverse y unirse a la Praja Mandal, de Junagadh. Para corroborar su lealtad a India, los líderes musulmanes declaraban que si India y Pakistán se enfrascaban en una guerra, los musulmanes de Junagadh pelearían contra Pakistán del lado de las fuerzas de la unión india. ${ }^{66}$ Diversos sectores de musulmanes se veían prácticamente obligados a dar demostraciones públicas de lealtad, de otra forma eran vistos con recelo por otros grupos sociales y aun por los mismos líderes políticos, como Vallabhbhai, cuyos llamados a la unidad para esta época mostraban una actitud cada vez más hostil hacia los musulmanes.

Después de la partición, Vallabhbhai no veía a los musulmanes como un grupo vulnerable. Siendo presidente del Comité de Minorías de la Asamblea Legislativa en 1949, Patel

${ }^{66}$ The Times of India, "Junagadh Muslims join Mandal. Decision at public meeting”, vol. cIX, núm. 280, 24 de noviembre de 1947, p. 7. 
mostraba abiertamente su desconfianza al afirmar que "los musulmanes de hoy son una minoría muy fuerte, unida y bien organizada. ¡Muy bien! Una minoría que puede forzar la partición del país no es una minoría en absoluto. ¿Por qué creen que son una minoría?". ${ }^{67}$ Con esto evidentemente minimizaba las condiciones adversas de este sector, además de que inhibía en otros la solidaridad o empatía por los problemas que esta comunidad, como las otras, enfrentaba. Aunque su postura fue endureciéndose y sus sentimientos nacionalistas hindúes se exacerbaron, Patel tuvo cuidado de distanciarse de organizaciones más bien fundamentalistas como el Rashtriya Swayamsevak Sangh, sobre todo después del asesinato de Gandhi, en 1948, a manos de uno de sus miembros. Así, discutía con Nehru sobre cómo poner mayores restricciones a esta organización para que sus actividades no fueran una amenaza a la paz y la tranquilidad de India. ${ }^{68}$

\section{Conclusiones}

Este artículo ha tratado de examinar la filosofía y la política de Gandhi y su percepción y aprehensión por uno de sus seguidores más importantes, Vallabhbhai Patel, quien fue una figura muy influyente y cuya aparente subordinación a Gandhi no evitó que tuviera un amplio margen para sus maniobras políticas. Su carrera política demostró su carácter como hombre de Estado y luchador social; no obstante, su nacionalismo anticolonial fue desplazado por una creciente sospecha hacia los musulmanes que lo condujo cada vez más lejos de Gandhi. Su propio contexto cultural, el devenir histórico y el activismo de las organizaciones hindúes detonaron este cambio en su percepción de los musulmanes, no como una minoría vulnerable sino como una amenaza para la unidad de India que tenía que demostrar fehacientemente su lealtad. A pesar de su cercanía con

${ }^{67}$ Vallabhbhai Patel, Sardar Patel. In Tune with the Millions, ed. G. M. Nandurkar y Manibehn Patel, parte II, vol. III, Ahmedabad, Sardar Vallabhbhai Patel Smarak Bhavan, 1976, p. 156.

${ }^{68}$ Vallabhbhai Patel, Sardar Patel: Select Correspondence, 1945-1950, ed. Das Durga, vol. vII, Ahmedabad, Navajivan Publishing House, 1973, p. 261. 
Gandhi y de una correspondencia con la filosofía del mabatma en el periodo de las satyagrahas (1918-1928), en Gujarat, Patel tuvo un pensamiento y métodos de acción independientes, si no es que opuestos a los de su mentor en distintos momentos. Su afiliación a las nociones gandhianas no fue un impedimento para que él también se vinculase a un movimiento hindú antiislámico. De aquí que no sean pertinentes los cuestionamientos que han planteado algunos estudiosos en épocas recientes acerca del porqué de las expresiones fundamentalistas hindúes en el "Gujarat de Gandhi". Como se ha explorado en este escrito, la ética de la no violencia gandhiana convivió con un nacionalismo hindú militante que fue ganando terreno aun entre los seguidores más cercanos del mahatma, quien, para la partición, ya había perdido influencia en las decisiones políticas. Así, la ideología hindú nacionalista pudo acomodarse y remontar en un contexto histórico que sentó precedentes de la cultura política del Gujarat e India contemporáneos.

Dirección institucional de las autoras:

Centro de Estudios de Asia y África

El Colegio de México

Camino al Ajusco 20

Pedregal de Sta. Teresa

10740, México, D.F.

$\triangle$ bmartinez@colmex.mx

凶ibanerje@colmex.mx

\section{Bibliografía}

Alavi, Hamza, "Ironies of History: Contradictions of the Khilafat Movement", en Mushirul Hasan (ed.), Islam, Communities and the Nation: Muslim Identities in South Asia and Beyond, Nueva Delhi, Manohar, 1998, pp. 25-56.

Amin, Shahid, "Gandhi como Mahatma: Distrito de Gorakhpur, UP Oriental, 1921-1922”, en Saurabh Dube (coord.), Pasados poscoloniales. Colección de ensayos sobre la nueva historia y etnografía de la India, México, El Colegio de México, 1999, pp. 345-401.

Chaterjee, Partha, "Gandhi and the Critique of Civil Society", en Ranajit Guha (ed.), Subaltern Studies III: Writings on South Asian 
History and Society, Nueva Delhi, Oxford University Press, 1984, pp. 153-195.

Cohen, Anthony P., The Symbolic Construction of Community, Londres, Routledge, 1989.

Gandhi, M. K., Hind Swaraj or Indian Home Rule, Ahmedabad, Navajivan Publishing House, 1938.

Gandhi, M. K., The Collected Works of Mahatma Gandhi, Nueva

Delhi, Ministry of Information \& Broadcasting, Government of India, 1970.

GandHI, Rajmohan, Patel. A Life, Ahmedabad, Navajivan Publishing House, 1990.

Hавів, Irfan, "Gandhi and the National Movement", Social Scientist, vol. 23, núm. 4, 1995, pp. 3-15.

Hardiman, David, Peasant Nationalists of Gujarat. Kheda District 1917-1934, Delhi, Oxford University Press, 1981.

Krishna, Balraj, India's Bismarck, Sardar Vallabhbhai Patel, Mumbai, Indus Source Book, 2007.

Kumar, Ravinder, "Class, Community or Nation? Gandhi's Quest for a Popular Consensus in India”, Modern Asian Studies, vol. 3, núm. 4, 1969, pp. 357-376.

Menta, Uday S., "Patience, Inwardness, and Self-Knowledge in Gandhi's Hind Swaraj”, Public Culture, vol. 23, núm. 2, 2003, pp. 417-429.

Menon, Vapal Pangunni, The Story of the Integration of the Indian State, Bombay, Orient Longman, 1969.

MukTA, Parita, "On The Political Culture of Authoritarianism", en Ghanshyam Shah, Mario Rutten y Hein Streefkerk (eds.), Development and Deprivation in Gujarat, Nueva Delhi-Thousand Oaks, Sage Publications, 2002, pp. 59-73.

Patel, Vallabhbhai, For a United India: Speeches of Sardar Patel, 19471950, Delhi, Publications Division, Ministry of Information and Broadcasting, Government of India, 1967.

Patel, Vallabhbhai, Sardar Patel. In Tune with the Millions, ed. G.

M. Nandukar y Manibehn Patel, parte II, vol. III, Ahmedabad, Sardar Vallabhbhai Patel Smarak Bhavan, 1976.

Patel, Vallabhbhai, Sardar Patel: Select Correspondence, 1945-1950, ed. Das Durga, vol. viI, Ahmedabad, Navajivan Publishing House, 1973.

Patel, Vallabhbhai, The Collected Works of Sardar Vallabhbhai Patel, ed. P. N. Chopra, vols. I y XII, Delhi, Konark Publishers, 19901999.

PRATT, Tim y James Vernon, “'Appeal from This Fiery Bed...': 
The Colonial Politics of Gandhi's Fasts and their Metropolitan Reception”, Journal of British Studies, vol. 44, núm. 1, 2005, pp. 92-114.

SHAH, Ghanshyam, "Traditional Society and Political Mobilization: The Experience of Bardoli satyagraha (1920-1928)", Contribution to Indian Sociology, vol. 8, núm. 89, 1974, pp. 89-107.

SkARIA, Ajay, "Immeasurable Equality: Gandhi and the Gift of Religion”, manuscrito inédito.

SKARIA, Ajay, "La religión de Gandhi", en Saurabh Dube e Ishita Banerjee (coords.), Otras modernidades: historias, culturas, identidades, México, El Colegio de México, 2011, pp. 159-178.

SPODEK, Howard, "Sardar Vallabhbhai Patel at 100", Economic Political Weekly, vol. 10, núm. 50, 1975, pp. 1925-1936.

STEIN, Burton, A History of India, Sussex, Willey-Blackwell, 2010.

Thapar, Romila, Somanatha: The Many Voices of a History, Nueva Delhi, Penguin, 2004.

The Bombay Chronicle, "Somnath", vol. xxxix, núm. 13, 12 de mayo de 1951, p. 4.

The Bombay Chronicle, “We'll hand over Junagadh to provisional government alone' Sardar's reply to Pakistan: Token referendum favours Indian Union”, vol. xxxv, núm. 268, 15 de noviembre de 1947, p. 9.

The Times of India, "Junagadh Muslims join Mandal. Decision at public meeting", vol. cIX, núm. 280, 24 de noviembre de 1947, p. 7.

The Times of India, "Pakistan's interference in Junagadh; Sardar Patel's criticism”, vol. cIX, núm. 273, 15 de noviembre de 1947, p. 9.

Vaghela, Arun, Anvesna Itihasna Lekhono Sangrah, Gandhinagar, Gujarat Sahitya Akademi, 2001.

Valiani, Arafaat, Militant Publics in India. Physical Culture and Violence in the Making of a Modern Polity, Nueva York, PalgraveMacmillan, 2011.

YAGNIK, Achyut y Suchitra Sheth, The Shaping of Modern Gujarat: Plurality, Hindutva and Beyond, Nueva Delhi, Penguin, 2005.

Young India (1919-1931), "M. K. Gandhi, 'Prohibition Campaign"”, vol. XI, 23 de mayo de 1929, p. 171.

ZAKARIA, Rafiq, Sardar Patel and Indian Muslims. An Analysis of his Relations with Muslims before and after India's Partition, Bombay, Bharatiya Vidya Bhavan, 1996. 
